\title{
Congenital arterioportal fistula of the liver with reversal of flow in the superior mesenteric vein
}

\author{
J. Bakker ${ }^{1}$, S. G. F. Robben ${ }^{1}$, F. W. J. Hazebroek ${ }^{2}$, M.Meradji ${ }^{1}$ \\ ${ }^{1}$ Department of Pediatric Radiology, Sophia Children's Hospital, Dr Molewaterplein 60, NL-3015 GJ Rotterdam, The Netherlands \\ ${ }^{2}$ Department of Pediatric Surgery, Sophia Children's Hospital, Dr Molewaterplein 60, NL-3015 GJ Rotterdam, The Netherlands
}

Received: 25 January 1994/Accepted: 10 February 1994

\begin{abstract}
We report on a newborn with a large arterioportal fistula of the liver complicated by a sudden reversal of flow in the portal and superior mesenteric vein, leading to congestion and ischemia of the small intestine. Ultrasound, duplex Doppler sonography and angiographic features are presented. Ligation of the hepatic artery led to a complete recovery.
\end{abstract}

Intrahepatic arterioportal fistulas (APFs) in childhood are rare [1-3]. In childhood APFs are usually regarded as congenital; later in life they are more often of traumatic or iatrogenic origin. Complications of an APF are acute or chronic portal hypertension and high-output cardiac failure.

We present a patient with a large arterioportal fistula of the liver complicated by acute portal hypertension. Reversal of flow was demonstrated by duplex Doppler sonography and confirmed by angiography.

\section{Case report}

A 3-week-old male infant was referred to a local hospital having suffered bilious vomiting and bloody diarrhea for 3 days. Pregnancy and delivery had been uncomplicated. Two days after admission the clinical situation suddenly deteriorated. Referral to our hospital for further evaluation and treatment followed.

On admission the infant was dehydrated and convulsive with poor circulation. Physical examination of the abdomen revealed distention, little peristaltic movement and a souffle over the right upper quadrant. The liver edge was palpable $5 \mathrm{~cm}$ below the right costal margin. Intubation and mechanical ventilation were required. Laboratory tests were as follows: $\mathrm{Na} 121 \mathrm{mmol} / \mathrm{l}, \mathrm{Hb}$ $10.0 \mathrm{mmol} / 1$, Hct $0.49 \mathrm{l} / 1, \mathrm{~S}_{\mathrm{a}} \mathrm{O}_{2} 87 \%$, pH 7.17, $P \mathrm{CO}_{2} 44.3 \mathrm{~mm} \mathrm{Hg}$, $\mathrm{BE}-11.2$. Abdominal radiographs showed a large liver, distended bowel loops, thickened intestinal walls and a few air-fluid levels. On the chest radiograph moderate cardiac enlargement, elevated diaphragm and increased pulmonary vascular markings were seen.

Ultrasound examination demonstrated a wide right hepatic artery directly entering the right portal vein. Duplex Doppler sonography showed reversal of flow in the portal and superior mesen-

Correspondence to: S. G.F. Robben teric vein, being hepatofugal instead of hepatopetal (Fig. 1). The character of flow was arterial instead of venous. Hepatic veins, inferior vena cava and right atrium were normal. The wall of the small intestine was thickened and peristaltic movement was diminished. Cardiac sonography showed dilatation of the left ventricle with decreased contractility. Abdominal angiography confirmed the findings of the ultrasound study (Fig. 2). The large right hepatic artery entered an aneurysmatic system. Venous outflow was through the dilated right portal vein, with retrograde filling of the superior mesenteric vein. No varicose abnormalities of the esophagus or the intestine were seen.

At surgery an arteriovenous fistula between the hepatic artery and the portal vein was found. Organs distal to the celiac axis were poorly perfused. The small bowel and its mesentery were congested, cyanotic and atonic. Temporary occlusion of the large common hepatic artery improved the color of the bowel and the thrill over the APF in the liver disappeared. The common hepatic artery was then ligated just distal to the cystic artery. The postoperative course was uneventful. Ultrasound of the abdomen showed no flow in the APF. The superior mesenteric vein was still somewhat wide but the flow was in a hepatopetal direction (Fig. 3). After 6 months sonography demonstrated further normalization of the situation; complete resolution of the aneurysmatic system had occurred. Five years after surgery the patient was in good health without signs of abdominal bruit, portal hypertension or malabsorption.

\section{Discussion}

Arterioportal fistula (APF) is a well-known disorder in adults. It can be secondary to trauma, hepatocellular carcinoma, liver cirrhosis or aneurysms, or it can be of iatrogenic origin. In adults the most common complication is chronic portal hypertension with its sequelae. APFs in children are very rare. Only three pediatric cases of congenital origin have been described [1-3]. Acute portal hypertension was the first symptom in two of these cases, leading to distended abdomen, vomiting and diarrhea $[1,2]$. Other complications are high-output cardiac failure and hypoperfusion of organs distal to the APF.

Our patient presented with signs of acute portal hypertension. Prominent features were abdominal distention, persistent vomiting, bloody stools and eventually cardiac and respiratory failure. Three factors contributed 

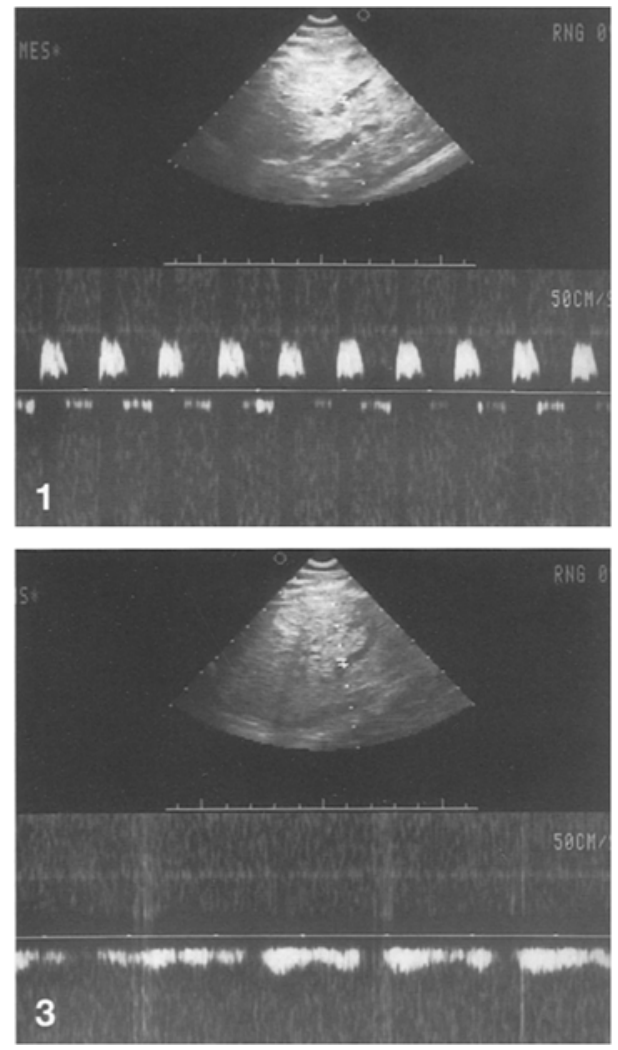
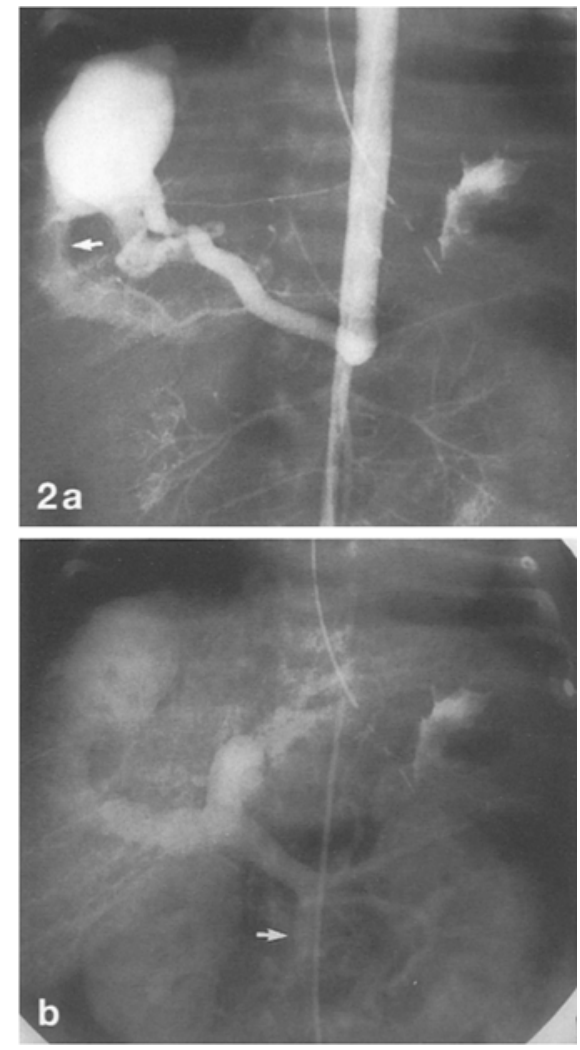

Fig. 1. Sagittal scan of superior mesenteric vein. Pulsed Doppler sonography shows pulsatile flow displayed above the baseline, indicating retrograde flow

Fig. 2. a Aortic angiography, arterial phase. Dilated hepatic artery feeding an aneurysmatic fistula drained by a dilated right portal vein (arrow). Note the small diameter of the aorta and its branches distal to the celiac trunk. b Aortic angiography, venous phase. Retrograde flow in common portal vein, superior mesenteric vein (arrow) and splenic vein

Fig. 3. Postoperative sagittal scan of superior mesenteric vein. Pulsed Doppler sonography shows normal continuous forward flow, displayed below the baseline to the poor circulation of the small bowel. Firstly, reversal of flow in the portal and superior mesenteric vein led to congestion. Secondly, a steal phenomenon occurred: the high flow in the hepatic artery compromised blood flow distal to its origin. Finally, dehydration caused the mesenteric circulation to deteriorate even further.

A hypothesis for the acuteness of onset of symptoms is given by Helikson et al. [2]. Delayed closure of the ductus venosus could explain the symptom-free interval after birth. During the first weeks of life, flow through the APF may be sufficiently strong to prevent closure of the ductus venosus, which temporarily acts as a collateral channel. Final closure of the ductus leads to a redirection of flow into the portal system, resulting in severe mesenteric vascular congestion.

Helikson used angiography to demonstrate an APF with retrograde flow in the portal and superior mesenteric vein, resulting in a congested and cyanotic small bowel [2]. Von Stiglbauer et al. described the use of duplex Doppler sonography to demonstrate APF and reversal of flow in the portal vein in adults [4]. Reversal of flow in the superior mesenteric vein caused by APF and demonstrated by duplex Doppler sonography has, to our knowledge, never been reported. Other causes of retrograde flow in the portal vein in children are severe cirrhosis, veno-occlusive disease [5] and Budd-Chiari syndrome.

Duplex Doppler sonography or color-coded sonography is the modality of choice to evaluate flow direction and flow pattern of APFs. It is a non-invasive, lowcost investigation without risk from ionizing radiation and is preferable to angiography where apparent reversal of flow can be an artifact due to forceful injection of contrast medium [6, 7]. However, if therapeutic intervention is imperative angiography is still necessary, either for preoperative planning or to evaluate the possibility of embolization.

\section{References}

1. Grybosky JD, Clemett A (1967) Congenital hepatic artery aneurysm with superior mesenteric artery insufficiency: a steal syndrome. Pediatrics 39:344-346

2. Helikson MA, Shapiro DL, Seashore JH (1977) Hepatoportal arteriovenous fistula and portal hypertension in an infant. Pediatrics 60: 921-924

3. Inon AE, DÁgostino D (1987) Portal hypertension secondary to congenital arterioportal fistula. J Pediatr Gastroenterol Nutr 6: $471-473$

4. Von Stiglbauer R, Hübsch P, Gritzmann N (1988) Zur Diagnose arterioportaler Kurzschlußverbindungen der Leber mittels gepulster und farbkodierter Doppler-Sonographie. Fortschr Röntgenstr 149: 214-215

5. Kriegshauser SJ (1988) Hepatic veno-occlusive disease after bone marrow transplantation: diagnosis with duplex sonography. AJR 150: 289-290

6. Takayasu K, Takashi M, Musha H (1982) Spontaneous reversal or portal blood flow demonstrated by percutaneous transhepatic catheterization: report of two cases. Gastroenterology 82: 753757

7. Ohnishi K, Saito M, Sato S (1985) Direction of splenic venous flow assessed by pulsed Doppler flowmetry in patients with a large splenorenal shunt. Gastroenterology 89: 180-185 